ROCZNIKI HUMANISTYCZNE

Tom LXIX, zeszyt 5 - 2021

DOI: http://doi.org/10.18290/rh21695-12

URSZULA TOPCZEWSKA

\title{
WANN WIRD SCHWEIGEN ZUM SPRECHAKT? ILLOKUTIONEN IM GRENZBEREICH SPRACHLICHEN HANDELNS
}

\begin{abstract}
A b stract. Ziel des vorliegenden Beitrags ist es, zu zeigen, dass Schweigen im Gespräch ein indirekter Sprechakt im Sinne von Searle sein kann. Die vielfältigen semiotischen Funktionen von Sprechpausen, die in diesem Beitrag vorgestellt werden, legen nahe, dass es sich um ein unabhängiges Segment in einer Reihe von Äußerungen handelt, und somit um einen Sprechakt, der von den vorangehenden und folgenden verbalen Handlungen getrennt ist. Seine Interpretation hängt von Konventionen ab, die allerdings im Gegensatz zu direkten Sprechhandlungen selten ausschließlich symbolische Verfahren hervorrufen. In den meisten Fällen lösen Sprechpausen auch ein von Keller definiertes symptomatisches Interpretationsverfahren aus, das zu indirekten Schlussfolgerungen über die Kommunikationsabsichten der Sprecher führt.
\end{abstract}

Schlüsselwörter: Stille; Sprechpause; Illokution; indirekter Sprechakt.

Ziel des vorliegenden Beitrags ist es, nachzuweisen, dass Schweigen als indirekter Sprechakt aufgefasst werden kann. Konversationsanalytische Untersuchungen zu seiner Bedeutung (z.B. Saville-Troike, Tiersma, Sifianou, Kurzon, Jaworski, Ephratt, Nakane, Grimmwood und Miller, Pietikäinen) haben aufgezeigt, dass Schweigen in Gesprächen semantisch eine hochkomplexe Struktur darstellt. Es ist eindeutig mehr als ein suprasegmentales Merkmal von Sprechakten, insofern es intentional eingeführt bzw. zugelassen wird. Die darzustellenden vielfältigen semantischen Funktionen des Schweigens legen die These nahe, dass Schweigen ein selbständiges Segment in einer Äußerungsreihe ist und somit einen von der vorangehenden bzw. der nachfolgenden verbalen Handlung getrennten Sprechakt darstellt. Seine Interpretation beruht auf Konventionen, die allerdings seltener sym-

Dr habil. URsZUla TOPCZEWSKA - Universität Warschau, Institut für Angewandte Linguistik; Korrespondenzadresse: ul. Dobra 55, 00-312 Warszawa; e-mail: u.topczewska@uw.edu.pl; ORCID: https://orcid.org/0000-0001-7010-6570. 
bolische Verfahren - wie bei direkten Sprechakten - evozieren, sondern viel häufiger ein symptomatisches Interpretationsverfahren im Sinne Kellers veranlassen, wenn von Sprechpausen auf kommunikative Intentionen des Sprechers geschlossen wird.

\section{WAS KANN OHNE WORTE GESAGT WERDEN?}

Sprechen ist nicht immer nötig und nicht immer ausreichend, um eine kommunikative Intention auszudrücken. Zum einen gibt es Fälle erfolgreicher wortloser Kommunikation, wie etwa im folgenden Beispiel von Watzlawick, Beavin und Jackson, in dem eine verbale Handlung gänzlich unterlassen wird und trotzdem ein intensiver Gedankenaustausch stattfindet:

The man at a crowded lunch counter who looks straight ahead, or the airplane passenger who sits with his eyes closed, are both communicating that they do not want to speak to anybody or be spoken to, and their neighbors usually ,get the message" and respond appropriately by leaving them alone. This, obviously, is just as much an interchange of communication as an animated discussion. (Watzlawick, Beavin und Jackson 51-52)

Zum anderen können vielsagende Pausen auch in Konversationen vorkommen und die gesprochenen Worte hervorheben, begleiten bzw. ganz ersetzen. Mit Schweigen (statt mit einem Oh Gott!) kann auf eine schockierende Nachricht reagiert werden, etwa wenn jemand über eine schwere Krankheit, einen schweren Unfall oder einen Todesfall berichtet. Beispiele für Situationen, in denen Schweigen als Redebeitrag interpretiert wird, sind ebenso: „the tense silence at the beginning of an academic seminar when no student wants to answer the tutor's question; or the moments of anticipation when an interlocutor hesitates to respond to a complex question" (Grimmwood und Miller 77).

Es gibt sogar Situationen, in denen Schweigen die einzige akzeptable Antwort im Gespräch ist, z.B. wenn jemand einen schmutzigen Witz auf Kosten einer anwesenden Person macht und man weder das Gesicht des Witzerzählers noch das der betroffenen Person verletzen will. In diesem Fall ist Schweigen nicht nur ein natürliches Symptom der Scham (wie etwa das Rotwerden), sondern zugleich ein intentionaler Akt (Willensakt) des Gesprächspartners. Seine Bedeutung ergibt sich aus der sozialen Konvention, dass selbst in einer peinlichen Situation eine direkte Konfrontation aus Höflichkeitsgründen vermieden wird. 
Mit recht wenigen Ausnahmen, wie etwa Schweigen als Symbol für Gedenken und Trauer in einer Schweigeminute oder Schweigen, nachdem man solche Ausdrücke wie ... Punkt, ... und damit basta gehört hat, steht Schweigen nicht symbolisch für eine Sprecherintention, sondern drückt sie symptomatisch aus. Symptome sind Ergebnisse menschlicher Handlungen, zu deren Interpretation nur die Relation der Kausalität notwendig ist (vgl. Keller 123). Solange Schweigen als Symptom für kommunikative Intentionen interpretiert wird, kann man es auf unterschiedliche, u.U. sogar gegensätzliche Ursachen zurückführen, was nicht selten ein Missverständnis der Sprecherintention bewirkt und insbesondere in der interkulturellen Kommunikation schwerwiegende Folgen haben kann. Ein Beispiel hierzu aus dem Leben türkischer Kinder in Deutschland, die streng in der muslimischen Tradition erzogen werden:

Ein Vater, der mit seinem Kind schimpft, erwartet, dass das Kind schweigt und erträgt. Fragen sind immer rhetorisch und dürfen nicht beantwortet werden. Es macht gar nichts, wenn das Kind genervt guckt, aber es darf die Autoritätsperson nicht anschauen. Verhält sich dasselbe Kind aber einem deutschen Lehrer gegenüber genauso - es guckt genervt, schweigt, wenn es gefragt wird, schaut den Lehrer nicht an - findet der das Verhalten respektlos. Und das Kind versteht gar nicht, was die Lehrer von ihm wollen." (Sadigh)

Eine schweigend geäußerte Mitteilung ist allerdings auch nicht viel weniger eindeutig als Mitteilungen, die durch verbale Äußerungen gemacht werden, zumal das, was Menschen mit Hilfe von Worten tun (z.B. Sätze S im Kontext $\mathrm{K} \mathrm{zu}$ benutzen, um auszusagen, dass $\mathrm{p}$ ), nicht identisch zu sein braucht mit dem, was die Worte selbst symbolisieren. Beim verbalen Kommunizieren werden sprachliche Mittel beinahe regelmäßig dazu verwendet, indirekt dem Kommunikationspartner etwas zu verstehen zu geben. Als Beispiele indirekter verbaler Kommunikation seien hier folgende Gespräche angeführt:

(1) A: War Franz-Josef Strauß katholisch oder evangelisch?

B: Er war Bayer! (vgl. Keller 203)

(2) A: Kannst du kochen?

B: Ich bin Franzose. (vgl. Recanati 5)

B's Gesprächsbeiträge sind keine direkten Antworten auf die jeweils gestellte Frage. Wenn aber B davon ausgeht, dass A weiß, dass Bayern typischerweise katholisch sind bzw. Franzosen typischerweise gut kochen, kann 
er auch davon ausgehen, dass seine jeweiligen Gesprächsbeiträge im gegebenen Kontext ausreichend informativ sind. Das geteilte Wissen (B weiß, dass A weiß, dass B weiß, dass p) der Gesprächsteilnehmer ermöglicht es, die kommunikative Intention des Sprechers zu bestimmen.

Indirekte Sprechakte zeichnen sich nach Searle dadurch aus, dass die angezeigte (sekundäre) Intention nicht mit der intendierten (primären) Handlungsintention übereinstimmt, wobei die letztere unter Bezugnahme auf die erstere erschlossen wird. Von einem Sprecher, dessen kommunikative Intention nicht problemlos zu erkennen ist, wird man üblicherweise mit Warum sagst du das jetzt? eine Klärung fordern. Denn mit einer Äußerung, der zwar eine Bedeutung, aber kaum ein kommunikativer Sinn zugeschrieben werden kann, geben wir uns meist nicht zufrieden. Sie wird vielmehr als versteckte und somit indirekte Drohung, Warnung, Kritik usw. verstanden (vgl. Auer 79). Dieses kommunikative Verhalten gilt auch für die Interpretation des Schweigens: Wir interpretieren es je nach Kontext als Zustimmung, Verneinung, Ablehnung, Widerspruch usw.

\section{SCHWEIGEN ALS SPRECHHANDLUNG}

Unter Schweigen verstehe ich im Folgenden Sprechpausen, die mindestens 1 Sekunde andauern und aufgrund des turn-taking-Mechanismus den Gesprächspartner nicht zur Übernahme der Rednerrolle veranlasst haben. Gemeint sind also nicht die intermediären bzw. finalen Segmentierungspausen oder die gefüllten bzw. ungefüllten Verzögerungspausen zur Wortsuche, die im Gespräch kaum intentional vorkommen. Beim Schweigen handelt es sich um sog. Relevanzpausen (Zifonun et al. 244). Diese werden intentional eingesetzt und haben eine semiotische Funktion, z.B.

(3) A: So I was wondering would you be in your office on Monday?

$(2.0)^{1}$

A: Probably not.

E: $\mathrm{Hmm}$ yes $=^{2}$

A: $=$ You would?

E: Ya

A: Could you give us ten minutes of your time? (zit. nach Levinson 348)

\footnotetext{
${ }^{1}$ GAT-Notation für gemessene Pausen (Angabe in Sekunden mit einer Stelle hinter dem Punkt).

${ }^{2}$ Ein Gleichheitszeichen markiert einen schnellen Einstieg in einen neuen Gesprächszug.
} 
Die Sprechpause tritt hier statt einer verbalen Äußerung auf und wird aufgrund der Konventionen des Sprecherwechselsystems als ein Redebeitrag von Sprecher E interpretiert. Einen deutlichen Hinweis darauf, wie das Schweigen interpretiert wurde, liefert der dem 2 Sekunden langen Schweigen folgende Beitrag von Sprecher A: Das Schweigen wird von ihm als eine abschlägige Antwort auf seine eingangs gestellte Frage interpretiert. Die dabei wirkende Interpretationskonvention legt Levinson wie folgt aus: Weniger bevorzugte Teile von Paarsequenzen sind z.B. durch Zögern gekennzeichnet; das Schweigen kann also "als Vorbote einer weniger bevorzugten Antwort“ (Levinson 348) ausgelegt werden. Da die weniger bevorzugte Antwort im Kontext einer Bitte die Verneinung ist, vermutet A, dass das Schweigen seines Gesprächspartners eine verneinende Antwort einleitet. Die nächsten Redebeiträge E's korrigieren allerdings diese Interpretation; das Schweigen erhält also seine endgültige Bedeutung aus dem gesamten Gesprächskontext heraus.

Eine andere Interpretationskonvention wird in Beispiel (4) eingesetzt, in dem eine Mutter ihr Kind auffordert, ihr die Uhrzeit zu nennen. Im Kontext einer Abfragung wird das Schweigen als „Ich weiß es nicht“" interpretiert, was auch die nächsten, immer einfacheren Fragen der Mutter bestätigen.

(4) M: What's the time by the clock?

M: What's the time?

(3.0)

M: Now what number's that?

R: Number two

M: No it's not

What is it?

R: It's a one and a nought (zit. nach Levinson 355)

Beim Schweigen werden keine Worte, wohl aber Intentionen geäußert, und genau in diesem Sinne kann eine Sprechpause vielsagend sein und als Sprechhandlung gelten. Schweigend kann man bitten, auffordern, fragen, widersprechen, beleidigen, warnen, bejahen oder verneinen. Man kann mit Hilfe von Schweigen u.U. auch etwas versprechen, wenn Schweigen z.B. in Antwort auf einen Vorschlag als Zustimmung interpretierbar ist. Wer sich schweigend äußert, weiß in der Regel auch, mit welchen Folgen er/sie zu rechnen hat. In Gerichtsprotokollen wird das Schweigen der an einer Verhandlung Beteiligten ebenso wie ihre Redebeiträge verzeichnet und beim Urteil über die Schuld oder Unschuld des Angeklagten mit berücksichtigt (vgl. Tiersma). 


\section{INTENTIONALES SCHWEIGEN}

Woran erkennt man, dass die Unterlassung einer Sprechhandlung selbst eine Sprechhandlung ist? ${ }^{3}$ Wenn im Gesprächskontext Hinweise dafür identifiziert werden können, dass eine Sprechpause bewusst eingesetzt wurde, kann angenommen werden, dass sie auch intentional ist. Es gibt allerdings auch eine weitere Auffassung der Intentionalität, die von Searle vor dem Hintergrund seiner Sprechakttheorie entwickelt wurde. Searle lässt bei verbalen Sprechhandlungen auch unbewusste Intentionen zu und erklärt allein die Interpretierbarkeit einer Handlung als im gegebenen Kontext zweckmäßig zur Bedingung ihrer Intentionalität. Alle als intentional interpretierbaren, d.h. nach sozial geltenden Regeln und Konventionen verlaufenden Sprechhandlungen dürfen als intentional angesehen werden, zumal die meisten Intentionen nicht der theoretischen, sondern der praktischen (also kaum bewusst erlebten) Intelligenz entstammen (vgl. Searle, Minds, Brain, and Science 65).

Insofern Schweigen nicht ausschließlich von externen Faktoren determiniert ist, sondern sich weitgehend aus den mentalen Zuständen der Handelnden, v.a. ihrer Kenntnis sozialer Konventionen, ableiten lässt und die gegebene Handlungsintention von ihnen auch jederzeit bewusst gemacht werden kann, kann in Anschluss an Searle vom Schweigen als intentionalem Sprechakt gesprochen werden. Die Schwächen dieser Intentionalitätsauffassung liegen aber auf der Hand: Searles Ansatz gründet in der Annahme, dass Sprechakte intentionale Zustände ausdrücken, deren Intentionalität eine sekundäre (not intrinsic) ist. Sie bedeuten etwas kraft der Tatsache, dass ihnen aufgrund der kollektiven Intentionen, der sog. We-intentions von den Sprechern eine Bedeutung zugeschrieben wird; daher muss nicht das Bewusstsein, sondern die sich aus den jeweiligen $\mathrm{We}$-intentions ergebende Proposition als Bedingung für das Vorhandensein einer kommunikativen Intention angesehen werden. Dem Schweigen als Ausdruck intentionaler Zustände liegt aber genau genommen keine Lokution und somit auch keine Proposition zugrunde. Schweigen kann trotzdem auf Handlungsintentionen und somit indirekt auch auf mentale Zustände und propositionale Bedeutungen referieren.

Problematisch ist die Annahme einer Proposition als Interpretationsbasis für indirekte Sprechakte bereits bei manchen verbalen Handlungen, wie etwa Fragen (vgl. Hanks). Wird ihre illokutionäre Kraft als Funktion F der Satzbedeutung $\mathrm{p}$ aufgefasst, wie es Searle tut, müsste man die wahrheitsfunktio-

\footnotetext{
${ }^{3}$ Auf die Korrelation zwischen Intentionalität und Informativität hat Jaszczołt (139) hingewiesen
} 
nale Satzbedeutung auch bei nicht indikativen Sätzen identifizieren können oder konsequenterweise annehmen, dass die letzteren ebenso wie nonverbale Handlungen keine illokutionäre Kraft besitzen. Dies steht im offensichtlichen Widerspruch mit den alltäglichen Kommunikationserfahrungen: Gerade Fragen und Aufforderungen stellen typische Illokutionen dar.

Nach Austin können auch nonverbale Mittel zur Ausführung eines illokutionären oder perlokutionären Sprechakts genutzt werden. Austin erwägt insbesondere Gesten als intentionales Handeln, erwähnt aber auch schweigende Zustimmung oder nicht verbal geäußerte Versprechen. Sowohl für die verbalen als auch für die nonverbalen Sprechakte gilt laut Austin, dass sie sich interaktiv ereignen. Eine illokutionäre Handlung gilt erst dann als tatsächlich ausgeführt, wenn sie für die Rezipienten wahrnehmbar und interpretierbar ist (vgl. Austin 116). Ein gelungener Sprechakt muss als solcher verstanden werden, allerdings ist das Vorhandensein einer Lokution keine notwendige Bedingung dafür, dass ein illokutionärer Akt geglückt ist, d.h. verstanden wurde. Denn Austin sieht ein, dass der Wahrheitswert logischer Sätze, der für die lokutionäre Bedeutung ausschlaggebend ist, kaum alle Bedeutungsaspekte umfasst. Es gibt Kontexte, in denen gerade die Illokution viel wichtiger oder sogar hinreichend für die Bestimmung einer Proposition sein kann. Dies ist beispielsweise der Fall, wenn der Sprecher auf eine leere Klasse von Gegenständen referiert und der lokutionäre Akt überhaupt nicht zustande kommt (vgl. Topczewska 72).

Als weiterer Beweis dafür, dass eine Lokution keine notwendige Grundlage für eine Illokution sein muss, dienen Austin Fälle, in denen wir z.B. keinen Zweifel darüber haben, dass eine Handlung die Sprechhandlung der Aufforderung war, uns aber nicht sicher sind, was genau damit gefordert wurde, d.h. welche Proposition damit mitgeteilt wurde (vgl. Austin 114115). Notwendig für das Verstehen eines illokutionären Sprechakts sind also lediglich bestimmte Interpretationskonventionen, kraft derer auch nonverbale Kommunikationsmittel bedeutsam erscheinen und zum Durchführen von Illokutionen genutzt werden können. Die Konventionen verhelfen dazu, dass die illokutionäre Funktion eines Sprechakts regelmäßig - und nicht etwa zufällig - auf bestimmte Sprecherintentionen zurückgeführt wird. Wenn man also in der Tradition Austins die Bedeutung eines Sprechakts nicht mehr von seiner Proposition abhängig macht, sondern sie in die illokutionäre Kraft verlegt, muss man auch Schweigen als „extremly powerful“ (Grimmwood und Miller 77) anerkennen, selbst wenn es in vielen Fällen semantisch ambivalent bleibt. 


\section{SCHWEIGEN ALS INDIREKTER SPRECHAKT: DAS BEISPIEL TRUDEAU}

Beim täglichen Corona-Briefing am 3. Juni 2020 wurde der kanadische Premier Justin Trudeau nach seiner Meinung zum Umgang des US-Präsidenten mit den Demonstranten gefragt, die im Zusammenhang mit dem Mord an George Floyd gegen Rassismus protestierten. Der fragende Journalist wollte wissen, was Trudeau darüber denke, dass gegen Demonstranten Tränengas eingesetzt worden sei, um dem Präsidenten einen Fototermin zu ermöglichen, und dass Trump selbst einen Armeeeinsatz gegen Demonstranten fordere. Für den Fall, dass Trudeau das Verhalten des USPräsidenten nicht würde kommentieren wollen, fragte der Journalist, welche Botschaft er damit senden würde. Der Journalist wörtlich:

You've been reluctant to comment on the words and actions of the U.S. president. But we do have Donald Trump now calling for military action against protesters, we saw protesters tear-gassed yesterday to make way for a presidential photo-op - I'd like to ask you what you think about that and if you don't want to comment, what message do you think you're sending? (URL1)

Trudeaus Antwort war zunächst ein 21 Sekunden langes Schweigen, während dessen er mehrmals zum Sprechen anzusetzen schien. Daraufhin verzichtete er sowohl auf einen Kommentar zum Verhalten des US-Präsidenten als auch auf eine Beantwortung der Frage, welche Botschaft er damit der Öffentlichkeit sende, sondern sagte nur:

We all watch in horror and consternation what's going on in the United States. It is a time to pull people together, but it is a time to listen. It is a time to learn what injustices continue despite progress over years and decades. But it is a time for us as Canadians to recognize that we, too, have our challenges, that black Canadians and racialized Canadians face discrimination as a lived reality every single day. There is systemic discrimination in Canada, which means our systems treat Canadians of colour, Canadians who are racialized differently than they do others. It is something that many of us don't see, but it is something that is a lived reality for racialized Canadians. We need to see that, not just as a government and take action, but we need to see that as Canadians. We need to be allies in the fight against discrimination. (URL2)

In den Leserkommentaren zu Trudeaus Schweigen auf Spiegel Online ${ }^{4}$

\footnotetext{
${ }^{4}$ Die Kommentare sind unter folgendem Link abrufbar URL3.
} 
überwiegen zwei Interpretationen: Zum einen wird - wie in Beispiel (5)-(7) - hervorgehoben, dass Trudeau schweige, um zu zeigen, dass er sich von Trump distanziere: Er denke nach, bevor er etwas sage, sei also nicht so wie der „Krawallmacher“ Trump. Zum anderen wird betont, dass das Schweigen inszeniert (nicht spontan, also nicht ehrlich) sei. Diese Tatsache wird entweder positiv wie in (8) oder negativ wie in (9) bewertet. Im letzteren Fall sei das, was Trudeau schließlich sagt, enttäuschend, denn er kritisiere Trump nicht klar, sondern gebe eine ausweichende Antwort auf die ihm gestellte Frage.

(5) Die USA sind ein grosser Handelspartner Canadas - Schweigen ist damit die höflichste Form der Ablehnung.

(6) Weil es andeutet, daß es eine Menge zu Trump zu sagen gäbe, was er aber - weil er, anders als Trump, ein Staatsmann ist, nicht sagt.

(7) Eine Meisterleistung! Denn er weiß genau dass der Kollege weiter südlich nicht in der Lage ist mehr als zwei Sekunden zu schweigen.

(8) Ein tolles Statement! Ich glaube zwar, dass Frage und Antwort inszeniert sind, siehe seltsame Frage: ... und wenn sie nicht kommentieren möchten, welche Botschaft senden sie damit? Und Trudeaus schon vorheriges Starren in die Kamera.

(9) Was für ein Poser!

In jedem Fall ist Trudeau ein Populist, der von der ganzen Sache profitieren möchte.

Vielleicht hatte das Schweigen nur die Funktion, die Spannung auf die folgende Sprechhandlung zu steigern und damit ihre Aussage als im Kontext besonders relevant hervorzuheben. Auch dann wird es aber als intentional aufgefasst:

(10) Die lange Schweigepause war für sich schon eine subtile Botschaft.

(11) Premierminister Trudeau ist in den meisten Fällen sehr gut vorbereitet, wenn er etwas sagt. Und so, wie er es dann formuliert, muss man davon ausgehen, dass er diese Antwort, diese kurze Rede, bereits im Kopf vorbereitet hatte. Er hätte also nicht zögern müssen. Ebenso weiß Justin Trudeau sehr genau um die Effekte von Sprache, Betonung, und Pausen. Deshalb gehe ich davon aus, dass das lange Schweigen ein bewusstes Zeichen war. Vielleicht die beste Art, dem Krawallmacher im Süden von Kanada zu antworten.

Interessanterweise wird in zwei der insgesamt 82 Kommentare vermutet, Trudeaus Schweigen sei nur einer technischen Panne geschuldet. In diesem Fall müsste es als peinliches Missgeschick interpretiert werden, zumal es viel zu lange andauert, um es als typische Verzögerungspause zur Wortfindung interpretieren zu können: 
(12) Irgendwie scheint es mir nicht unwahrscheinlich, das, so konzentriert er in die Kamera schaut, das er tatsächlich auf Text auf dem teleprompter wartet .. und was sich seine Textschreiber auf die schnelle als passende Replik einfallen lassen. So ganz scheint mir das nicht das Gesicht eines Menschen, der nach Worten sucht.

Dass Trudeau die Pause benötigte, um nach einer passenden Antwort auf die Frage des Journalisten zu suchen, ist durchaus denkbar. Allerdings ist dabei auch zu berücksichtigen, dass ein so erfahrener Politiker wie Trudeau im Umgang mit Medien gründlich geschult ist und die ihm gestellte Frage zu erwarten war, insofern Donald Trump mit seiner Erklärung, das Militär gegen Demonstranten einsetzen zu wollen, einen massiven Tabubruch begangen hatte. Die Antwort kann also kaum eine Ad-hoc-Antwort gewesen sein.

Das Kommunikationsmuster Frage-Antwort dient im politischen Kontext oft dazu, eine Auskunft zu den Themen zu erhalten, die die Öffentlichkeit gerade bewegen. Der antwortende Politiker zielt dabei darauf ab, die Antwort zur positiven Selbstdarstellung zu nutzen oder zumindest eine negative Außenwirkung zu vermeiden (vgl. Klein 367). Trudeau hat sich im Laufe seiner politischen Karriere immer wieder gegen Rassismus und Diskriminierung engagiert, aber ein kanadischer Premierminister müsste dennoch besonderen Wert auf gute Beziehungen zu den USA legen und sowohl aus wirtschaftlichen als auch aus rein diplomatischen Gründen darauf achten, dass er mit seiner Antwort weder die USA angreift noch der Öffentlichkeit eine missverständliche Botschaft vermittelt. Die Frage des Journalisten stellte ihn also vor ein Dilemma: sich von Trump zu distanzieren, ohne ihn dabei direkt anzugreifen, oder der Öffentlichkeit seine Sympathie für die Black-LivesMatter-Bewegung klarzumachen, ohne diese Sympathie direkt auszudrücken.

Zusammenfassend lässt sich festhalten: Trudeaus Mimik während des Schweigens vermittelt den Eindruck, er müsse um die passenden Worte ringen. Dieser Eindruck kann als Symptom unterschiedlicher kommunikativer Intentionen interpretiert werden, z.B.:

1. Trudeau zögert mit seiner Antwort aus diplomatischen Gründen und wägt seine Worte ab, um mit seiner Stellungnahme Trump nicht anzugreifen.

2. Sein Schweigen ist ein rhetorisches Stilmittel, um bestimmte Effekte bei der Öffentlichkeit zu erreichen.

3. Trudeaus Antwort verzögerte sich wegen einer technischen Panne, und unter diesen Umständen wollte er lieber abwarten als seine Rede zu improvisieren. 
Doch unabhängig davon, auf welche Ursache Trudeaus Schweigen zurückgeführt wird, weist jede der oben angeführten Interpretationen darauf hin, dass das Schweigen einen kommunikativen Zweck hatte: Entweder wollte der kanadische Premier eine echte Kritik an Trump indirekt äußern, oder er tat so, als ob er sie äußern wollte, oder er wollte zumindest mit der Antwort auf die ihm gestellte Frage zögern.

Die Einordnung von Trudeaus Schweigen als intentional setzt nicht unbedingt seine vorherige Planung voraus, sondern die Intentionalität ist in diesem Fall schon dadurch gegeben, dass Trudeau sich bewusst war, dass das Schweigen als zweckmäßig interpretiert werden würde. Ob Trudeaus Schweigen eine zuvor gewählte diskursive Strategie war oder nicht, hat letztendlich kaum Einfluss auf die Tatsache, dass bei seiner Interpretation eine Intentionalität vorausgesetzt wird.

\section{SCHLUSSBEMERKUNGEN}

Schweigen bedeutet wörtlich genommen ein Fehlen des Gesagten; gebraucht man es aber intentional, hat es zwar nach wie vor keine wörtliche, aber doch eine illokutionäre Bedeutung. Selbst ein intentionales Schweigen drückt nicht direkt eine wahrheitswertkonditionale Proposition aus, und es braucht auch keine solche als Illokutionsbasis zu haben, denn es ist seine illokutionäre Kraft, aus der sich aus seine Bedeutung ergibt.

In der Regel drückt Schweigen eine kommunikative Intention nicht symbolisch aus, sondern die ihm zugrundeliegende Intention wird symptomatisch interpretiert. Die symptomatische Interpretation beruht darauf, aus der wahrgenommenen Sprecherhandlung und ihrem Kontext kausale Schlüsse zu ziehen, d.h. vom Kommunikationsmittel Schweigen auf den Zweck des Schweigens zu schließen. In den Spiegel-Online-Kommentaren zum Schweigen Trudeaus wird das Schweigen beispielsweise als Mittel interpretiert, mit Hilfe dessen Trudeau den Zweck erreicht, Kritik an Trump indirekt auszudrücken bzw. diese Kritik zu inszenieren.

So wie im symbolischen Interpretationsverfahren das Gemeinte erschlossen werden soll, so gilt es im Falle der symptomatischen Interpretation den relevanten Grund für das Schweigen zu finden und somit den Zweck der Unterlassung einer verbalen Kommunikation zu erkennen. Die Interpretation des relevanten Zwecks setzt ein bestimmtes Hintergrundwissen voraus. In dem analysierten Fall gehört zu diesem Wissen etwa die Kenntnis der politi- 
schen Einstellung Trudeaus, der wirtschaftlichen Interessen Kanadas und nicht zuletzt der öffentlichen Rezeption politischer Aussagen eines Regierungschefs. Wenn bei den Kommunikationspartnern markante Differenzen in diesem Wissen vorliegen, weichen dementsprechend auch ihre Interpretationen des Relevanten in einem Sprechakt voneinander ab.

\section{LITERATUR}

Auer, Peter. Sprachliche Interaktion. Eine Einführung anhand von 22 Klassikern. Niemeyer, 1999.

Austin, John L. How to do things with words. The William James Lectures delivered at Harvard University in 1955. Clarendon Press, 1962.

Ephratt, Michal. "The functions of silence”. Journal of Pragmatics 40, 2008, S. 1909-1938.

Grimwood, Tom, u. Paul K. Miller. "How to Do Things without Words". J.L. Austin on Language, hrsg. von Brien Garvey, Palgrave Macmillan, 2014, S. 70-85.

Jaszczołt, Katarzyna. "Pomiędzy semantyką a pragmatyką". Metodologie językoznawstwa. Podstawy teoretyczne, hrsg. von Piotr Stalmaszczyk, Wydawnictwo Uniwersytetu Łódzkiego, 2006, S. 131-154.

Jaworski, Adam. "Silence and small talk". Small Talk, hrsg. von Justine Coupland, Pearson Education, 2000, S. 110-132.

Keller, Rudi. Zeichentheorie. Zu einer Theorie semiotischen Wissens. Francke, 1995.

Klein, Josef. "Sprache in der Politik". Handbuch Pragmatik, hrsg. von Fank Liedtke und Astrid Tuchen, J.B. Metzler Verlag, 2018, S. 358-369.

Kurzon, Dennis. Discourse of Silence. John Benjamins, 1998.

Levinson, Steven C.. Pragmatik. Niemeyer, $2000^{3}$.

Nakane, Ikuko. "The role of silence in interpreted police interviews". Journal of Pragmatics 43, 2011, S. 2317-2330.

Pietikäinen, Kaisa S. "Silence that speaks: The local inferences of withholding a response in intercultural couples' conflicts”. Journal of Pragmatics 129, 2018, S. 76-89.

Recanati, François. Literal Meaning. Cambridge University Press, 2004.

Sadigh, Parvin. „Allein zwischen Tradition und wahrem Leben“. Zeit Online 28. September 2011, https:/www.zeit.de/gesellschaft/familie/2011-09/migranten-jugendliche-bildung-integration/komplettansicht. Abruf am 31.05.2020.

Saville-Troike, Muriel. "The place of silence in an integrated theory of communication". Perspectives on Silence, hrsg. von Deborah Tannen und Muriel Saville-Troike. Ablex, 1985, S. 3-18.

Searle, John. Speech Acts. An Essay in the Philosophy of Language. Cambridge University Press, 1969.

Searle, John. "Indirect speech acts". Syntax \& Semantics, vol. 3: Speech Acts, hrsg. von Peter Cole und Jerry L. Morgan. Academic Press, 1975, S. 59-82.

Searle, John. Intentionality. An Essay in the Philosophy of Mind. Cambridge University Press, 1983.

Searle, John. Minds, Brain, and Science. Harvard University Press, 1984. 
Sifianou, Maria. "Silence and politeness". Silence. Interdisciplinary Perspectives, hrsg. von Adam Jaworski. Mouton de Gruyter, 1997, S. 63-84.

Tiersma, Peter. "The language of silence”. Rutgers Law Review 48, 1995, S. 1-99.

Topczewska, Urszula. Konnotationen oder konventionelle Implikaturen? Peter Lang, 2012.

URL1 = YouTube. https://www.youtube.com/watch?v=sjhF1GI9n8A. Abruf am 25.06.2020.

URL2 = YouTube. https://www.youtube.com/watch?v=sjhF1GI9n8A. Abruf am 25.06.2020.

URL3 = Der Spiegel. https://www.spiegel.de/politik/justin-trudeau-ueber-donald-trump-das-langeschweigen-a-4b2f0599-3a55-4707-b21a-4f31eb3d4558\#. Abruf am 25.06.2020.

Watzlawick, Paul, u. Janet H. Beavin, Don D. Jackson. Pragmatics of Human Communication A Study of Interactional Patterns, Pathologies and Paradoxes. Norton, 1967.

Zifonun, Gisela, u. Ludger Hoffmann, Ursula Brausse, Bruno Strecker, Joachim Ballweg. Grammatik der deutschen Sprache, vol. 3. Mouton de Gruyter, 1997.

\section{KIEDY MILCZENIE JEST AKTEM MOWY? ILLOKUCJE NA POGRANICZU DZIAŁAŃ JĘZYKOWYCH}

\section{Streszczenie}

Celem artykułu jest ukazanie milczenia jako niewerbalnego pośredniego aktu mowy. Wielorakie funkcje semiotyczne milczenia pozwalają traktować je jako samodzielny segment w ciągu wypowiedzeń, któremu odpowiada działanie odrębne od poprzedzającego i/lub następującego po nim działania werbalnego. Interpretacja milczenia rzadko jednak opiera się wyłącznie na symbolicznych procedurach interpretacyjnych; milczenie jest zwykle interpretowane nie jako działanie symboliczne, lecz jako symptom intencji komunikacyjnych mówcy, który zaniechał aktu werbalnego.

Słowa kluczowe: milczenie; pauza w wypowiedzi; illokucja; pośredni akt mowy.

\section{WHEN DOES SILENCE BECOME SPEECH ACT? ILLOCUTIONS ON THE BORDERLINE OF LINGUISTIC ACTION}

\section{Su m mary}

The aim of this paper is to show that silence in a conversation can be an indirect speech act in Searle's sense of the concept. The manifold semiotic functions of silence that will be presented in the paper suggest that it is an independent segment in a series of utterances, and thus constitutes a speech act separate from the preceding and following verbal acts. Its interpretation depends on conventions, but these conventions rarely evoke only symbolic procedures in contrast to direct speech acts. In most cases, pauses in speech also initiate a symptomatic interpretation procedure as defined by Rudi Keller, leading to indirect conclusions about the communicative intentions of the speakers.

Keywords: silence; speech pause; illocution; indirect speech act. 